\title{
Tumor-specific usage of alternative transcription start sites in colorectal cancer identified by genome-wide exon array analysis
}

\author{
Kasper Thorsen ${ }^{1}$, Troels Schepeler ${ }^{1}$, Bodil Øster ${ }^{1}$, Mads H Rasmussen', Søren Vang ${ }^{1}$, Kai Wang ${ }^{2,3}$, \\ Kristian Q Hansen ${ }^{1}$, Philippe Lamy ${ }^{1,4}$, Jakob Skou Pedersen ${ }^{1}$, Asger Eller ${ }^{1}$, Francisco Mansilla ${ }^{1}$, Kirsti Laurila ${ }^{5}$, \\ Carsten Wiuf ${ }^{4}$, Søren Laurberg ${ }^{6}$, Lars Dyrskjøt ${ }^{1}$, Torben F Ørntoft ${ }^{1}$ and Claus L Andersen ${ }^{1 *}$
}

\begin{abstract}
Background: Approximately half of all human genes use alternative transcription start sites (TSSs) to control mRNA levels and broaden the transcriptional output in healthy tissues. Aberrant expression patterns promoting carcinogenesis, however, may arise from alternative promoter usage.

Results: By profiling 108 colorectal samples using exon arrays, we identified nine genes (TCF12, OSBPL1A, TRAK1, ANK3, CHEK1, UGP2, LMO7, ACSL5, and SCIN) showing tumor-specific alternative TSS usage in both adenoma and cancer samples relative to normal mucosa. Analysis of independent exon array data sets corroborated these findings. Additionally, we confirmed the observed patterns for selected mRNAs using quantitative real-time reversetranscription PCR. Interestingly, for some of the genes, the tumor-specific TSS usage was not restricted to colorectal cancer. A comprehensive survey of the nine genes in lung, bladder, liver, prostate, gastric, and brain cancer revealed significantly altered mRNA isoform ratios for CHEK1, OSBPL1A, and TCF12 in a subset of these cancer types.

To identify the mechanism responsible for the shift in alternative TSS usage, we antagonized the Wnt-signaling pathway in DLD1 and LS174T colorectal cancer cell lines, which remarkably led to a shift in the preferred TSS for both OSBPL1A and TRAK1. This indicated a regulatory role of the Wht pathway in selecting TSS, possibly also involving TP53 and SOX9, as their transcription binding sites were enriched in the promoters of the tumor preferred isoforms together with their mRNA levels being increased in tumor samples.

Finally, to evaluate the prognostic impact of the altered TSS usage, immunohistochemistry was used to show deregulation of the total protein levels of both TCF12 and OSBPL1A, corresponding to the MRNA levels observed. Furthermore, the level of nuclear TCF12 had a significant correlation to progression free survival in a cohort of 248 stage II colorectal cancer samples.
\end{abstract}

Conclusions: Alternative TSS usage in colorectal adenoma and cancer samples has been shown for nine genes, and OSBPL1A and TRAK1 were found to be regulated in vitro by Wnt signaling. TCF12 protein expression was upregulated in cancer samples and correlated with progression free survival.

\section{Background}

Colorectal cancer $(\mathrm{CRC})$ is a leading cause of cancer mortality with more than 600,000 deaths per year globally [1]. CRC can be divided into two major subgroups: microsatellite stable (MSS) and microsatellite unstable cancers (MSI), the latter being characterized by a

\footnotetext{
* Correspondence: cla@ki.au.dk

'Department of Molecular Medicine, Aarhus University Hospital, Skejby, 8200 Aarhus N, Denmark

Full list of author information is available at the end of the article
}

defective mismatch repair system, which leads to mutations in microsatellite repeat regions [2]. The two subgroups also show differences in transcriptional profiles and clinical disease course [3]. A key event in the transformation of colonic epithelial cells is the activation of the Wnt-signaling pathway, which is observed in the vast majority of colorectal tumors [4]. Following activation of the Wnt pathway, $\beta$-catenin accumulates in the nucleus where it interacts with members of the TCF/LEF transcription factors, such as TCF1 (gene symbol TCF7) and

\section{Ciomed Central}


TCF4 (gene symbol TCF7L2) leading to expression of target genes including MYC, SOX9 and Cyclin D1. Furthermore, it has previously been shown that Wnt signaling also plays a regulatory role in alternative splicing $[5,6]$.

The generation of proteome diversity from a rather limited number of genes is primarily due to alternative splicing and alternative promoter usage, the latter leading to multiple transcripts from the same gene with different transcription start sites (TSSs). It has been estimated that $30-50 \%$ of all human genes have multiple promoters [7-9], and among the genes with experimentally well described alternative promoters are several cancer related genes such as MYC [10], TP53 [11] and BRCA1 [12]. Genome-wide predictive analysis has shown that alternative promoters are overrepresented in genes involved in development and transcriptional control, whereas genes with only a single promoter are more frequently involved in general cellular processes [9]. Alternative TSS can have crucial impact on both the transcriptional level and stability of the transcript as well as the function and cellular localization of the translated protein [8]. An example of a transcript with directly opposite effects is the transcription factor TCF1 where the use of alternative promoters results in the formation of two isoforms with different $\beta$-catenin binding capability. While the long isoform of TCF1 interacts with $\beta$-catenin and stimulates transcription of Wnt-target genes, the short isoform, unable to bind $\beta$-catenin but with the DNA binding domain intact, acts as a dominant negative regulator of $\beta$-catenin mediated Wnt signaling [13].

In the present study, a genome-wide search revealed nine genes with tumor-specific TSS usage in neoplastic colorectal tissue samples. For some of the genes, the tumor-specific TSS usage was not restricted to CRC, but was observed in other cancer types as well. The use of alternative TSSs in OSBPL1A and TRAK1 was found to be regulated by the Wnt pathway and also observed in gastric, prostate and brain tumors. Protein levels of OSBPL1A and TCF12 were found to be deregulated in colorectal tumors and the protein expression of TCF12 correlated with progression free survival of stage II CRC.

\section{Results}

Identification of differential transcription start site usage To investigate whether the use of alternative TSSs was associated with tumor development, normal colorectal mucosa $(n=24)$, colorectal adenoma $(n=49)$ and CRC samples $(n=35)$ were profiled using the Affymetrix Human Exon 1.0 ST Array. The Refseq database (hg18) was queried for genes with two or more known transcripts with different TSSs. In total, 2176 protein-coding genes containing multiple TSSs were identified and matched to exon array core transcript clusters by gene symbol of which 2036 genes had available exon expression data. Genes, containing exons potentially involved in tumor associated alternative TSS usage or alternative splicing, were identified using a splicing ANOVA approach. Additional filtering criteria (see methods) were used to select 156 candidate genes for manual inspection in a genomic context. The manual curation indicated tumor associated alternative TSS usage in nine (TCF12, OSBPL1A, TRAK1, ANK3, CHEK1, UGP2, LMO7, ACSL5 and $S C I N$ ) of the 156 genes. To confirm these findings, two independent CRC sample sets (covering a total of 18 normal mucosa, six adenomas and 28 CRC samples) were investigated. All nine genes showed expression profiles consistent with tumor-specific alternative TSS usage in at least one of the independent validation sets.

\section{qRT-PCR validation of alternative TSS candidates}

Three candidate genes (TCF12, OSBPL1A and TRAK1) with different expression characteristics were chosen for technical validation with quantitative real-time reversetranscription PCR (qRT-PCR) on a subset of the samples used for exon array analysis. Transcription factor 12 (TCF12), a member of the basic helix-loop-helix E-protein family with an E-box consensus binding site, exists as a long and a short variant. According to the exon array data, the long variant was expressed in both normal and tumor samples at roughly the same level, whereas the short isoform was expressed in the majority of the tumor samples, but not detected above background in the normal samples (Figure 1A). This indicated that the short isoform of TCF12 was de novo synthesized in the majority of the tumor samples, especially in adenomas and MSS carcinomas (Figure 1B). Exon array data from paired normal and adenoma samples were available for 18 patients, and for five of these patients exon array expression data were available for a matched carcinoma sample as well. Increased expression of TCF12 exon $1 \mathrm{~B}$ in tumor samples was seen in 21 of 23 sample pairs $(p=0.001)$ (Additional file 1A). For qRT-PCR validation of the shift in TCF12 TSS usage, six normal samples, 14 adenoma samples, divided in two groups based on expression of the short TCF12 isoform (high or low), and six MSS cancer samples were selected. qRT-PCR confirmed de novo expression of the short TCF12 isoform (exon 1B) in many tumor samples (Figure 1C) and showed strong correlation to the exon array data ( $R S=0.82$, Spearman correlation).

Oxysterol-binding protein-related protein 1 (OSBPL1A) is an intracellular lipid receptor which is a member of the oxysterol-binding protein (OSBP) family and exists as a long and a short transcript variant. The long variant was expressed at roughly the same level in normal and tumor samples, whereas the short variant was down-regulated in most tumor samples (Figure 1D). The ratio between the long and short variant was shifted significantly towards 


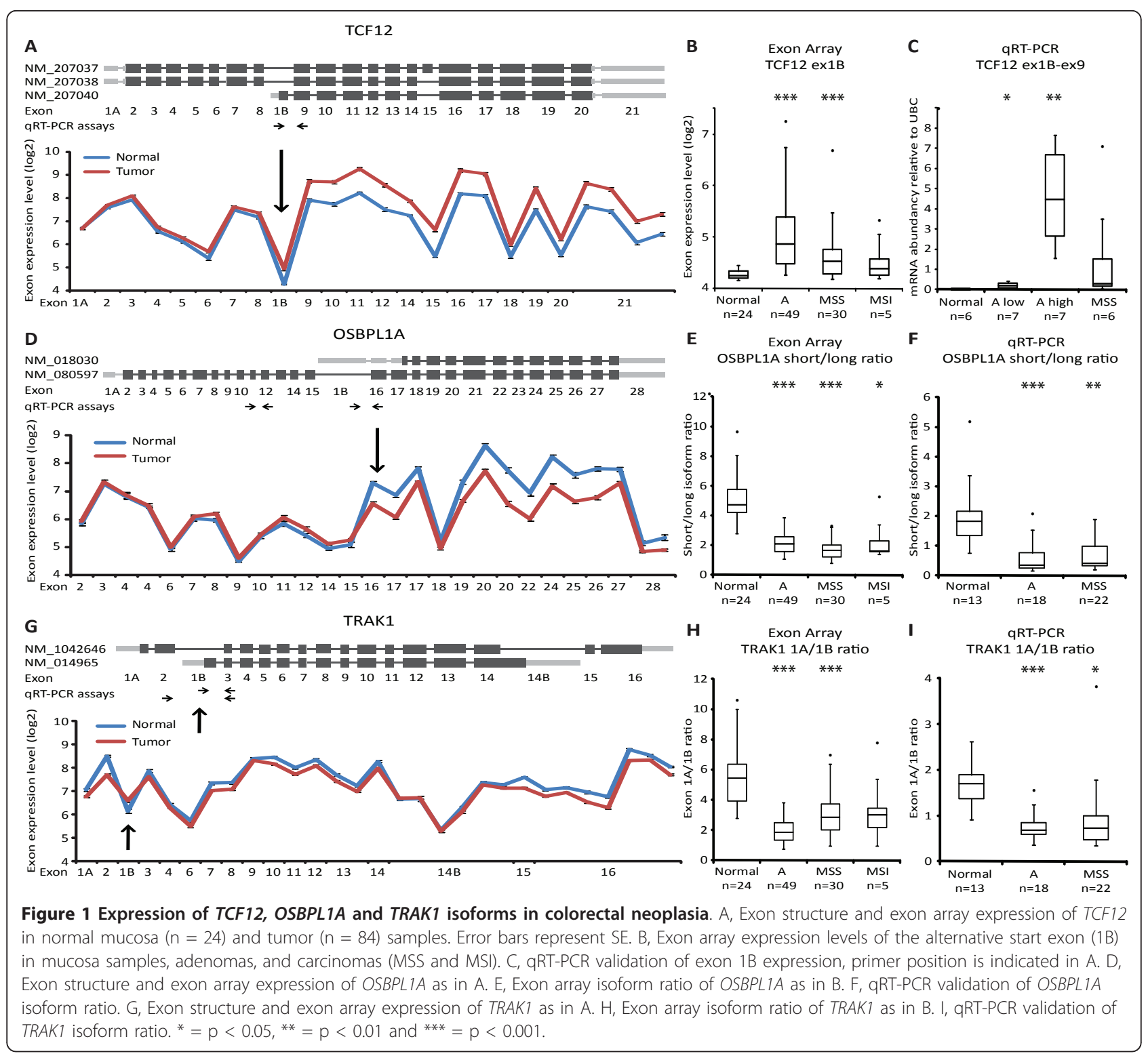

the long variant in both adenoma and cancer samples (Figure $1 \mathrm{E})$. This shift was also highly significant $\left(\mathrm{p}=7 \times 10^{-}\right.$ ${ }^{10}$ ) in the 23 paired normal and tumor samples, where a lower ratio was observed in all 23 tumor samples (Additional file 1B). This tumor-specific isoform shift was confirmed by qRT-PCR in 53 tissue samples (13 normal, 18 adenomas and 22 MSS cancers) (Figure 1F), which showed a robust correlation between exon array and qRT-PCR data ( $\mathrm{RS}=0.52$, Spearman correlation).

Finally, Trafficking kinesin-binding protein 1 (TRAK1), a kinesin-binding protein associated with mitochondria, contains two alternative start exons (exon $1 \mathrm{~A}$ and $1 \mathrm{~B}$ ) with differential expression in normal and tumor samples (Figure 1G). The ratio was shifted towards exon $1 \mathrm{~B}$ in both adenoma and cancer samples (Figure $1 \mathrm{H}$ ), including all 23 tumors with matched normal samples $\left(\mathrm{p}=5 \times 10^{-8}\right)$ (Additional file $1 \mathrm{C}$ ). This trend was also confirmed by qRT-PCR in the same 53 tissue samples described above (Figure 1I). Again, a fine correlation between exon array and qRT-PCR data was observed (RS $=0.58)$. We speculated whether alternative splicing rather than alternative TSS could explain the observed findings for TRAK1. However, in both normal and tumor samples only one qRTPCR product was observed for the primer set spanning the alternative TRAK1 exon $1 \mathrm{~B}$ (from exon 2 to 3 ), with a length consistent with lack of exon $1 \mathrm{~B}$. This demonstrates that no transcript containing exons $2,1 \mathrm{~B}$ and 3 exists. By contrast PCR with primers in exon $1 \mathrm{~B}$ and 3 produces a product. This indicates that the transcript template of the latter product starts with exon $1 \mathrm{~B}$, and, hence, that 
alternative TSS rather than alternative splicing is the mechanism generating the transcript. In conclusion, qRTPCR confirmed the tumor associated alternative TSSs for all three candidates, demonstrating that exon array data reliably identify alternative TSS usage.

\section{qRT-PCR validation of alternative TSS in laser capture microdissected samples}

It is well known that tissue composition changes from normal mucosa to tumor biopsies, and, potentially, the observed changes in TSS usage could reflect this rather than altered expression in the neoplastic cells. To address this question, we analyzed TCF12, OSBPL1A and TRAK1 isoform expression in laser capture microdissected (LCM) samples from six patients. From each patient, tumor and adjacent normal tissue biopsies were dissected to provide four samples per patient (normal epithelial cells, stroma from normal biopsies, CRC cells and cancer derived stroma). The short TCF12 isoform was expressed in half of the patients, and solely in cancer cells or, interestingly, cancer derived stroma, thus, confirming the tumor-specificity we observed in non-LCM samples (Additional file $2 \mathrm{~A}$ ). For OSBPL1A, a primer set measuring the long variant showed expression in half of the cancer and the cancer derived stroma samples, whereas the expression in the normal epithelium was low in all but one sample. A primer set measuring the expression of both isoforms showed low expression levels in both cancer epithelium and cancer derived stroma, whereas four of the six patients had a markedly higher expression in the normal epithelium and, for the same samples, a lower expression in the stroma from normal biopsies (Additional file 2B). TRAK1 exon 1A was found to be expressed in only two normal epithelial samples, whereas no expression of the tumor preferred exon $1 \mathrm{~B}$ was observed in either fraction from the normal sample. Exon 1B was expressed in half of the cancer epithelium samples of which two also had expression of exon 1A (Additional file 2C). In summary, the experiments confirmed that the tumor associated alternative TSS usages observed for TCF12, OSBPL1A and TRAK1 were indeed due to altered expression in the carcinoma cells, and not a consequence of altered tissue composition.

\section{Alternative TSS use in other cancer types}

Because many of the cancer associated expression changes identified to date generally play a role in cancers from different organs, we speculated whether the collection of tumor associated alternative TSSs identified here in the context of CRC could also have relevance to other cancers. To address this question, we acquired exon array expression data from gastric, liver, lung, bladder, brain and prostate normal and cancer samples. CHEK1 tumor associated alternative TSSs usage, which yields transcripts differing only in their 5 ' untranslated region (UTR) (Figure 2A), was most widely associated with cancer. In addition to CRC, a significant change in TSS usage was found in gastric, liver, lung and bladder cancer along with brain gliomas (Figure 2B-G). Significant expression changes of the OSBPL1A isoforms were observed in gastric and prostate cancer and metastases from prostate cancer patients along with brain gliomas (Additional file 3A-C). For liver, lung and bladder cancer, the trend was the same although not significant (Additional file 3D-F). Isoform expression changes were also found for TCF12 in brain gliomas (Additional file 3G). In summary, the analysis confirmed that some of the observed tumor associated TSS usages were not restricted to CRC.

\section{Transcription factor binding analysis}

Differences in preferred TSS could be explained by differential expression of transcription factors (TFs) controlling the activation of promoters located near the TSS of the nine selected genes. To identify TFs potentially responsible for the observed shifts in expressed isoforms, we performed TF binding site enrichment analysis in the TOUCAN2 software [14]. We chose to look at the sequence $300 \mathrm{bp}$ upstream and $100 \mathrm{bp}$ downstream from the TSS, which was defined as the start site of the matching Refseq sequence. Significantly overrepresented TF binding matrices were queried against the exon array expression data to identify deregulated TFs with enriched binding. Five TFs were significantly downregulated in tumor samples ( $p<0.05$, Benjamini-Hochberg corrected) and had enriched binding motifs near the TSS most commonly used in the normal mucosa samples (MEIS1, NR3C1, HNF4, IKZF3 and FOXO1), whereas only two TFs, TP53 and SOX9, were found to be significantly upregulated in tumor samples $(\mathrm{p}<0.05$, Benjamini-Hochberg corrected) along with having binding enrichment near the preferred TSS of the carcinoma samples, and, interestingly, SOX9 is known to be regulated by the Wnt pathway [15]. In summary, tumor-specific TF deregulation may contribute to the observed change of TSS usage.

\section{The Wnt pathway regulates the expression of OSBPL1A isoforms}

TCF4 is a key mediator of the Wnt-signaling pathway which is mutationally activated in $\sim 85 \%$ of CRCs [4]. Previously, in vivo TCF4 binding domains have been identified in the Ls174T CRC cell line using a ChIP-on-chip approach [16]. Interestingly, this study indicated that TCF4 binds just upstream of the TSS of the long OSBPL1A variant as well as upstream of both TRAK1 TSSs. This suggests that TCF4 is involved in regulating the TSS usage of these genes. To determine the impact of altered Wnt signaling (TCF4 activity) on the expression of OSBPL1A and TRAK1 isoforms, overexpression of a dominant negative form of TCF4 (dnTCF4) was used to interrupt 
A

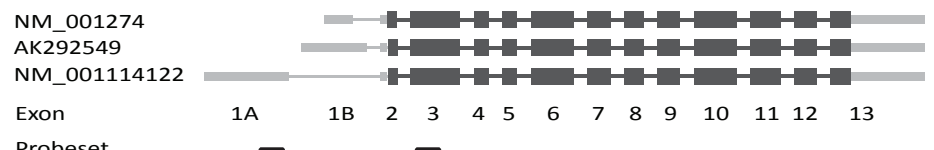

Probeset

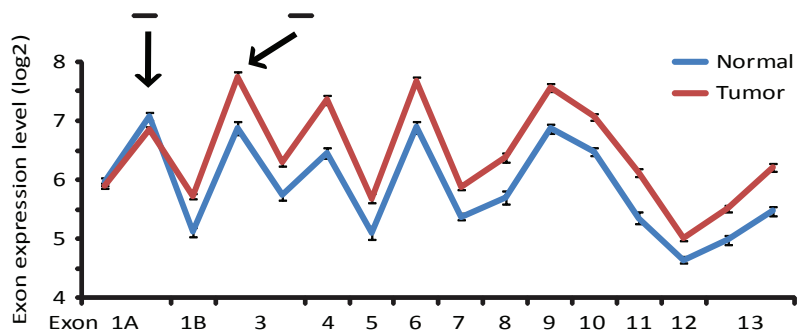

B

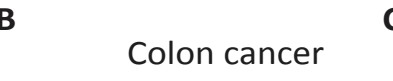

C Gastric cancer

D
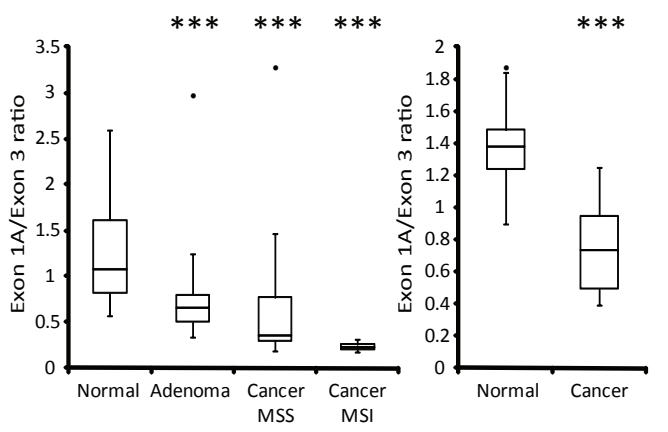

Liver cancer

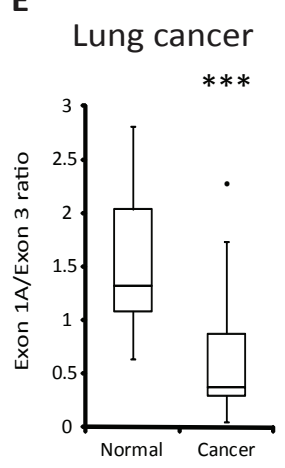

F

Bladder cancer

G
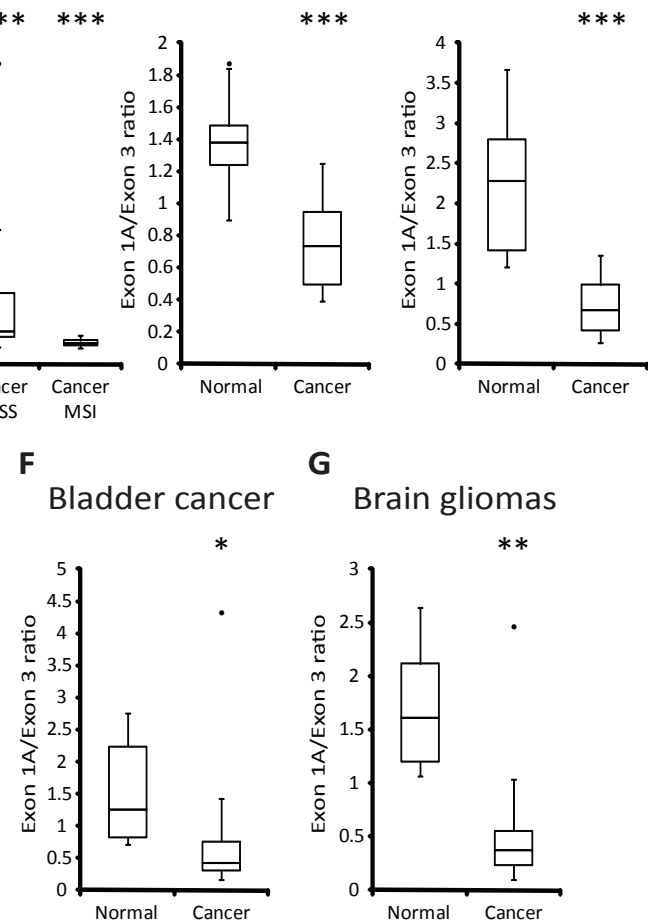

Figure 2 CHEK 1 isoform expression in colorectal and other cancers. A, Exon structure and exon array expression of CHEK1 in normal colorectal mucosa $(n=24)$ and colorectal tumor $(n=84)$ samples. Error bars represent SE. B, Exon array isoform ratio of CHEK1 in normal colorectal mucosa samples, adenomas, and carcinomas (MSS and MSI), C, expression in normal gastric and gastric cancer samples, D, expression in liver normal and cancer, E, expression in lung normal and cancer F, expression in bladder normal and cancer and, G, expression in normal brain and brain gliomas. ${ }^{*}=p<0.05,{ }^{*}=p<0.01$ and ${ }^{* *}=p<0.001$.

the TCF4 mediated part of the Wnt-pathway in the two CRC cell lines, DLD1 and Ls174T [17]. The ratio between the long and the short isoform of OSBPL1A was significantly changed in both the DLD1 $\left(\mathrm{p}=6.5 \times 10^{-4}\right)$ and the Ls174T $\left(p=5 \times 10^{-6}\right)$ cell lines when dnTCF4 was overexpressed (Figure 3A), mainly due to downregulation of the long variant. Interestingly, overexpression of a dominant negative form of TCF1, another transcription factor involved in mediating Wnt signaling, did not alter the ratio between the two OSBPL1A isoforms (Figure 3A). Although dnTCF4 targets the same sequence elements as TCF4, it cannot be ruled out that the observed expression changes could be unexpected artifacts of the dnTCF4 overexpression rather than disrupted Wnt-signaling. To address this caveat, we antagonized the signaling cascade upstream of TCF4 (in DLD1 cells) by siRNA mediated knock down of $\beta$-catenin, the key regulator of Wnt signaling. As for the dnTCF4 overexpression experiment, this 


\section{A}

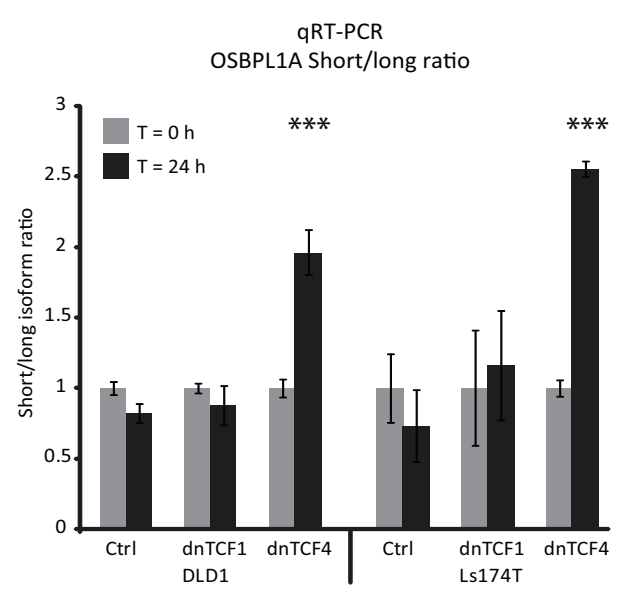

C

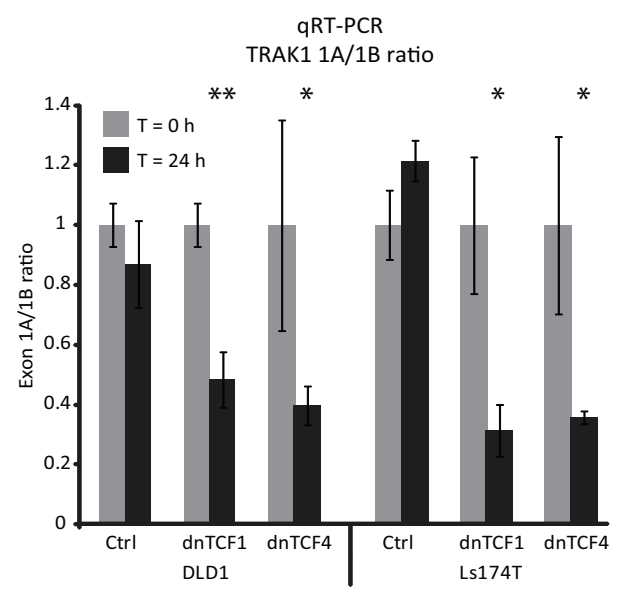

B

qRT-PCR

OSBPL1A Short/long ratio

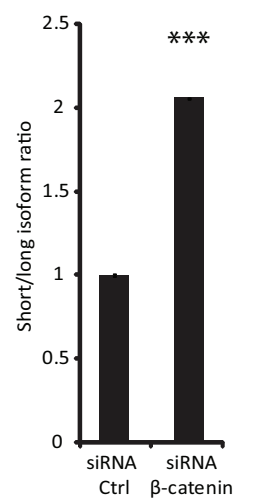

D

qRT-PCR TRAK1 1A/1B ratio

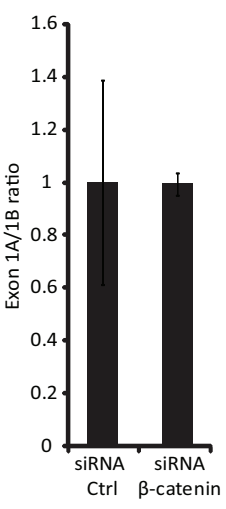

Figure 3 Wnt signaling regulates isoform expression of OSBPL1A and TRAK1. A, OSBPL1A isoform ratio measured by qRT-PCR in DLD1 and Ls174T cells expressing either dnTCF1 or dnTCF4 to disrupt the Wnt pathway. B, OSBPL1A isoform ratio in DLD1 cells transfected with either 20 nM control or $\beta$-catenin siRNA. C, TRAK1 exon 1A/1B ratio as described in A, D, TRAK1 exon 1A/1B ratio as described in $B .{ }^{*}=p<0.05$, ${ }^{* *}=p<$ 0.01 and ${ }^{* * *}=p<0.001$.

led to a significant change $\left(\mathrm{p}=2.9 \times 10^{-5}\right)$ in the ratio between the two OSBPL1A isoforms (with the same direction and magnitude, Figure $3 \mathrm{~B}$ ). This indicated that the Wnt pathway is truly involved in regulating the TSS usage of OSBPL1A.

For TRAK1, the ratio between the two isoforms shifted significantly, either when dnTCF1 or dnTCF4 was overexpressed (Figure 3C). Surprisingly, the most frequently found isoform in normal mucosa samples was the most infrequent when the Wnt-pathway was disrupted by the overexpression of dnTCF1 or dnTCF4. No change in the isoform ratio was seen when $\beta$-catenin was downregulated by siRNA (Figure 3D), implying that the change in TRAK1 ratio observed was executed in a $\beta$-catenin independent manner.
Immunohistochemical staining of TCF12 and OSBPL1A in matched normal and tumor tissues

To address whether the change in mRNA isoforms had impact on protein expression, immunohistochemical (IHC) analysis of TCF12 was performed on a tissue microarray (TMA) containing 50 normal mucosa and 51 matched adenocarcinoma samples with an antibody specific for an epitope present in both the long and short variant. The IHC revealed a significant increase in both the nuclear staining ( $\mathrm{p}<0.001$, Fishers exact test) and in the percentage of stained cell ( $p<0.001$, Fishers exact test) in cancer compared to normal samples (Figure 4A). This could be explained by the de novo synthesis of the short TCF12 isoform in a subset of cancers, as the mRNA encoding the long variant was expressed at equivalent 


\section{A}

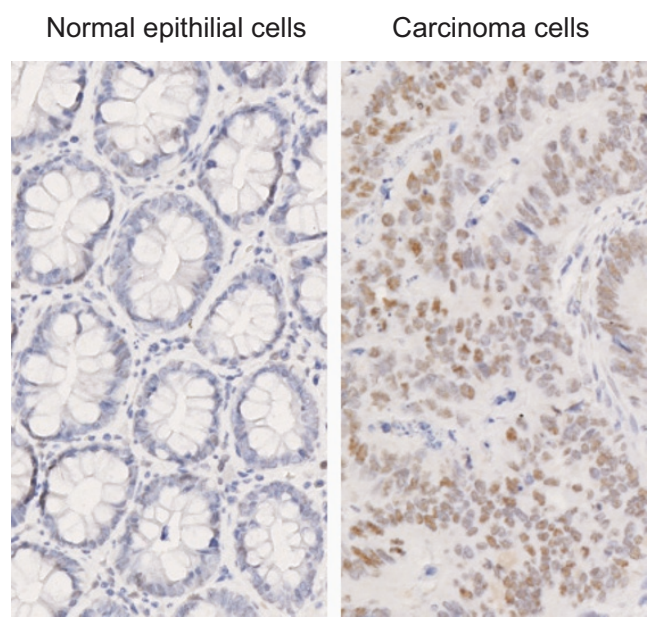

B

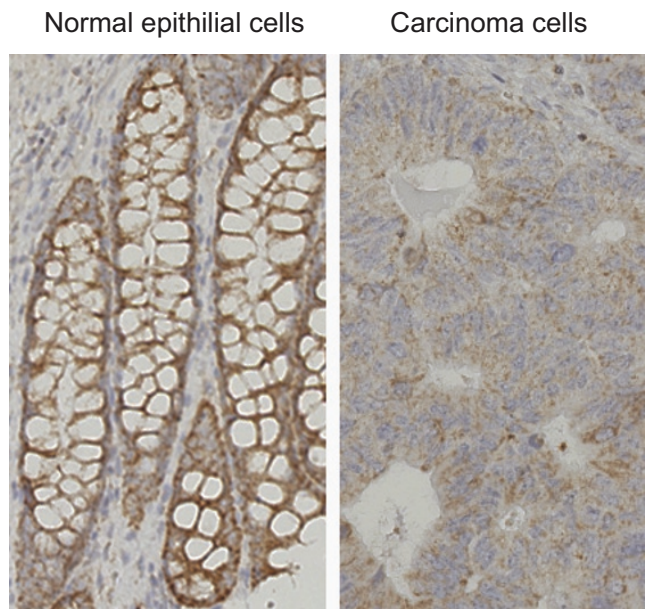

Figure 4 TCF12 and OSBPL1A expression detected by immunohistochemistry. A, TCF12 expression detected by $\mathrm{HC}$ in normal epithelial cells and adenocarcinoma cells. B, OSBPL1A expression detected by immunohistochemistry in normal epithelial cells and adenocarcinoma cells. All images are $\times 10$.

levels in normal and cancer samples. However, this may also reflect other different properties such as a difference in stability of the two isoforms. We observed only very few stained cells in the cancer stroma, corresponding to the weak expression of the short TCF12 mRNA isoform detected in LCM tumor stroma samples.

OSBPL1A IHC staining was performed on the TMA described above with an antibody targeting a peptide found in both transcript variants. This showed a significantly higher cytoplasmic staining intensity in normal epithelium compared to cancer cells $(\mathrm{p}<0.001$, Fishers exact test), whereas no difference in the percentage of stained cells was observed due to the pervasive expression of OSBPL1A in both normal and cancer tissue (Figure $4 \mathrm{~B})$. This was in agreement with our mRNA expression levels as the short OSBPL1A variant is downregulated in cancer samples, whereas the long OSBPL1A variant was expressed at similar levels in the normal and tumor samples.

\section{TCF12 protein expression is associated with recurrence- free survival}

To investigate the association between recurrence-free survival and TCF12 or OSBPL1A expression, we used a TMA containing biopsies from 268 stage II adenocarcinomas. Both the intensity of TCF12 and OSBPL1A and the percentage of stained cells were tested for association to recurrence-free survival. A very good inter-observer agreement was seen for all tested IHCs (kappa scores ranging from 0.74-0.83), and we found that TCF12 staining intensity was significantly associated with progression-free survival ( $\log$ rank test $\mathrm{p}=0.037)$ when data was dichotomized with the respect to strong intensity samples versus negative/weak/moderate intensity samples. Kaplan-Meier curves for TCF12 staining intensity (Figure 5) showed an increased recurrence rate for patients with biopsies that have a strong TCF12 staining. No significant association to recurrence-free survival was observed for the two OSBPL1A IHC staining parameters or the percentage of cells positive for TCF12. Univariate Cox regression analysis was implemented to examine the influence of each of the clinical parameters on progression-free survival, and we found TCF12 nuclear intensity to be significant $(\mathrm{p}=0.04)$ (Table 1). Finally, multivariate analysis was performed including TCF12 nuclear intensity and the number of lymph nodes examined, and we found TCF12 nuclear intensity to be independently associated with progression-free survival (Table 1).

\section{In silico protein predictions}

The potential structural and functional differences between the differentially expressed isoforms were analyzed using various in silico protein function and structure prediction tools, regulatory elements in untranslated regions (UTR) prediction tools along with already reported experimental data [18-24]. The results are summarized in Table 2, where the number of amino acids unique to the long or short variants is listed along with which protein domains are encoded by the unique amino acids, the presence of leader peptides and 5'UTR domains. Notably, significant functional differences are predicted between the long or short isoforms of TCF12, OSBPL1A, TRAK1, CHEK1, ANK3, LMO7 and SCIN indicating that the observed tumor associated changes in TSS usage probably have an impact on the growth properties of the neoplastic cells. 


\section{TCF12 nuclear intensity}

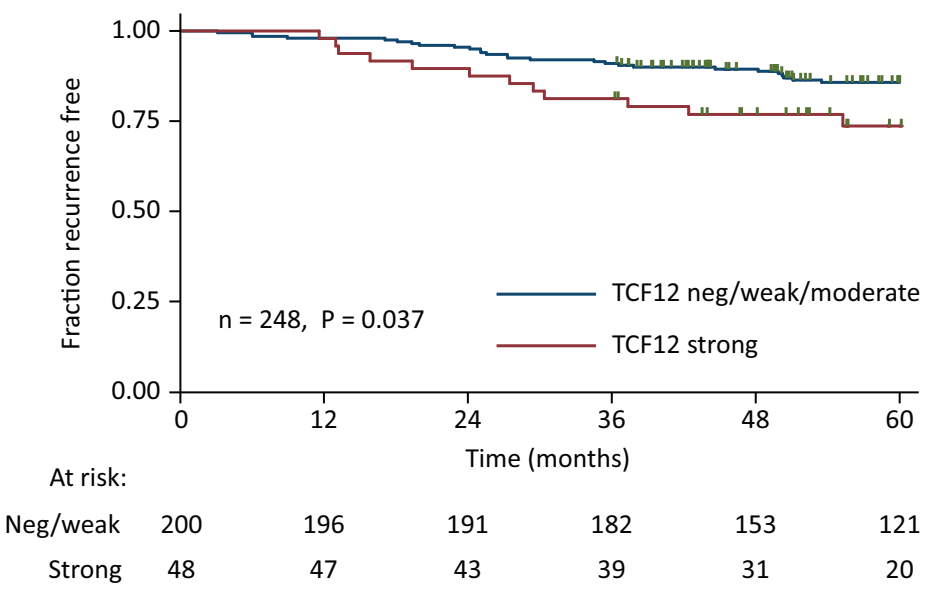

Figure 5 Kaplan-Meier curves for recurrence-free survival according to TCF12 staining intensity. IHC staining intensity of TCF12 on a colorectal stage II TMA was used to generate Kaplan-Meier curves for recurrence free survival. Intensity scores were divided in negative/weak/ moderate versus strong staining intensity. The log-rank test was used to generate the p-value by comparing the survival curves. Number at risk are listed below the plot and censored samples are indicated with a vertical bar.

\section{Discussion}

This study reports the results of a genome-wide screening for alternative TSS usage between normal and neoplastic colorectal samples. In total, we identified nine genes with alternative TSS usage and validated them in an independent cohort. A subset of the candidates was validated by qRT-PCR on the RNA used for array analysis and on RNA from LCM samples. TF binding analysis suggested that p53 and SOX9 might be responsible for altering the TSS use as they are both upregulated in cancer and have enriched binding sites in the promoters associated with the TSS preferred by the neoplastic cells. Disruption of Wnt signaling using two different CRC cell lines further showed that perturbed Wnt-signaling may also play a role in regulating alternative TSS usage of OSBPL1A and TRAK1, maybe through SOX9 which is known to be a Wnt-regulated TF [15].

The vast majority of scientific reports commonly refer to individual genes as one entity encoding a predominant transcript. This is a drastic oversimplification considering that experimental evidence supports the existence of at least two functional promoters for $>50 \%$ of all genes $[25,26]$, and, furthermore, it has the unfortunate consequence that potentially important transcriptional variants may be ignored. Such transcript diversity is generated by various mechanisms such as alternative splicing, alternative TSS usage and alternative polyadenylation usage, which generate families of transcripts from a single gene locus. Additionally, the use of DNA methylation appears to contribute to differential usage of alternative promoters in multiple tissue types [27] as well as in cancer [28]. We have previously detected cancer specific alternative splicing in colorectal, prostate and bladder cancer [29], and also shown that disruption of Wnt signaling can change the splicing pattern in colorectal, lung and gastric cancer [6]. The findings in this paper extend these previous observations by providing specific examples of transcript isoforms that arise through tumor-specific alternative TSS usage that occur not only in colorectal, but also in a wide range of other cancers. We have not addressed potential alternative splicing of TCF12, OSBPLA1 and TRAK1 in this study, but have shown that the exon expression pattern and $\mathrm{QPCR}$ validation is consistent with alternative TSS. In TCF12 and OSBPL1A, the expression follows the pattern of already described short and long transcript isoforms, and for alternative splicing to explain this pattern, novel extreme 5' start exons would have to be included in the transcript of both genes followed by alternative splicing of at least eight otherwise constituve spliced exons, a scenario we consider highly unlikely.

For the transcriptional changes to impact tumor cell growth, it is crucial that they translate into protein changes. We used two different strategies to address this issue. First, IHC analysis showed a significant difference in the total protein level of TCF12 and OSBPL1A between normal and cancer samples consistent with the mRNA expression pattern we observed. Furthermore, we found a correlation between the total TCF12 protein expression level and progression free survival. We did not perform IHC analysis of TRAK1 as a recent study already reported that elevated levels of TRAK1 are associated with poor prognosis in CRC patients [30]. Second, in silico analysis was used to predict the impact of an altered TSS on 
Table 1 Univariate and multivariate Cox regression analysis for progression free survival

\begin{tabular}{|c|c|c|c|c|c|c|c|}
\hline \multirow[b]{2}{*}{ Factor } & \multirow[b]{2}{*}{ Patients $(n=248)$} & \multicolumn{3}{|c|}{ Univariate analysis } & \multicolumn{3}{|c|}{ Multivariate analysis } \\
\hline & & HR & $95 \% \mathrm{Cl}$ & p-val & HR & $95 \% \mathrm{Cl}$ & p-val \\
\hline \multicolumn{8}{|l|}{ TCF12 expression } \\
\hline Neg/weak & 200 & 1 & Reference & & 1 & Reference & \\
\hline Strong & 48 & 2.03 & $1.03-4.01$ & 0.04 & 2.24 & $1.17-4.73$ & 0.02 \\
\hline \multicolumn{8}{|l|}{ Sex } \\
\hline Female & 120 & 1 & Reference & & & & \\
\hline Male & 128 & 1.82 & $0.95-3.51$ & 0.07 & & & \\
\hline \multicolumn{8}{|l|}{ Age at surgery } \\
\hline$<=71$ & 128 & 1 & Reference & & & & \\
\hline$>71$ & 120 & 1.26 & $0.67-2.38$ & 0.46 & & & \\
\hline \multicolumn{8}{|l|}{ Tumour location } \\
\hline Colon & 158 & 1 & Reference & & & & \\
\hline Rectum & 90 & 1.11 & $0.57-2.08$ & 0.80 & & & \\
\hline \multicolumn{8}{|l|}{ T-stage } \\
\hline pT3 & 226 & 1 & Reference & & & & \\
\hline pT4 & 22 & 0.81 & $0.27-2.80$ & 0.74 & & & \\
\hline \multicolumn{8}{|l|}{ Tumor size $e^{a}$} \\
\hline$<50 \mathrm{~mm}$ & 135 & 1 & Reference & & & & \\
\hline$>50 \mathrm{~mm}$ & 112 & 0.70 & $0.35-1.30$ & 0.24 & & & \\
\hline \multicolumn{8}{|l|}{ Histological type ${ }^{a}$} \\
\hline Adenocarcinoma & 217 & 1 & Reference & & & & \\
\hline Mucinous & 27 & 1.18 & $0.46-4.88$ & 0.50 & & & \\
\hline \multicolumn{8}{|l|}{ Number of nodes ${ }^{a}$} \\
\hline$<=10$ & 119 & 1 & Reference & & 1 & Reference & \\
\hline$>10$ & 119 & 2.01 & $1.00-4.04$ & 0.05 & 2.10 & $1.00-4.03$ & 0.05 \\
\hline
\end{tabular}

$\mathrm{HR}=$ hazard ratio; $95 \% \mathrm{Cl}=95 \%$ confidence interval.

${ }^{\text {ap }}$ atients with missing values were excluded from the analysis.

Table 2 Protein and 5' UTR in silico predictions

\begin{tabular}{|c|c|c|c|c|c|c|c|c|c|c|}
\hline \multirow[b]{2}{*}{ Gene } & \multicolumn{2}{|c|}{ Unique $A A^{a}$} & \multicolumn{2}{|c|}{ Protein domains } & \multicolumn{2}{|c|}{ Signal peptide } & \multicolumn{2}{|c|}{ Glucosylation and phosphorylation } & \multicolumn{2}{|c|}{$\underline{5^{\prime} \mathrm{UTR}}$} \\
\hline & Long & Short & Long & Short & Long & Short & Long & Short & Long & Short \\
\hline TCF12 & 193 & 23 & & & Yes & Yes & 10 glyco, 27 phos & & & \\
\hline OSBPL1A & 513 & & 3 Ank $^{\mathrm{b}}$,Pleck ${ }^{\mathrm{c}}$ & & No & No & 31 phos & & & \\
\hline TRAK1 & 96 & 38 & & & Yes & No & & & & \\
\hline CHEK1 & 0 & & & & No & No & & & $\mathrm{uORF}{ }^{\mathrm{d}}$ & \\
\hline ANK3 & 872 & 6 & $20 \mathrm{Ank}^{\mathrm{b}}$ & & No & No & 3 glyco, 33 phos & & & \\
\hline UGP2 & 11 & & & & No & No & & & & $\mathrm{uORF}^{\mathrm{d}}$ \\
\hline LMO7 & 285 & & Calponin & & Yes & No & 21 phos & & IRES & \\
\hline ACSL5 & 56 & & & & No & Yes & & & & \\
\hline SCIN & 247 & & 2 Gelsolin & & No & No & & & & $\mathrm{uORF}^{\mathrm{d}}$ \\
\hline
\end{tabular}

\footnotetext{
${ }^{\mathrm{a}} \mathrm{AA}=$ Amino acids

${ }^{\mathrm{b}}$ Ank $=$ Ankyrin repeats

${ }^{\mathrm{C}}$ Pleck $=$ Pleckstrin

${ }^{d} \mathrm{uORF}=$ Upstream open reading frame

${ }^{\mathrm{e}} \mathrm{RRES}=$ Internal ribosome entry site
} 
protein function, and we found that OSBPL1A has 31 phosphorylation sites and protein interaction domains that are unique to the long isoform, indicating a loss of function for the short isoforms. The long TCF12 variant encodes 10 glycosylation and Yin-Yang sites along with 27 phosphorylation sites not present in the short isoform. However, both isoforms contain DNA binding and dimerization domains, indicating separate functions of the isoforms, and not a simple dominant negative function of the short isoform. Indeed, both the long and short isoform of the TCF12 have previously been described as having distinct functions in thymocyte development $[31,32]$. Deregulation of $T c f 12$ has recently been causally implicated in CRC in a DNA transposon based forward genetic screen in a mouse model. Interestingly, in this study, Tcf12 was the fourth most commonly mutated gene locus ( $A p c$ being the most commonly mutated gene) [33]. This clearly indicates that TCF12 could have an important effect in CRC development. In silico predictions for the remaining candidate genes indicated diverse functional differences such as altered protein localization, changes in protein-protein interaction domains and differences in translational regulation and efficiency.

The selective use of alternative TSSs has been observed in different cell types, tissue types and developmental stages [8], however, the mechanism regulating the alternative TSS usage is often less well described. Aberrant TSS usage has also been linked to cancer, and in vitro studies of hypoxia in a CRC cell line revealed hundreds of genes with altered TSSs [34]. To elaborate on the possible mechanisms regulating the alternative TSS usage in CRC, we combined TF binding site enrichment analysis and TF expression analysis and showed that several TFs were dysregulated in tumors along with having enriched binding sites in the TSS regions. Furthermore, we used cell line models to demonstrate that abrogation of Wnt signalling leads to altered TSS usage for OSBPL1A and TRAK1, showing that the major pathway in CRC development could be a key regulator of TSS usage.

\section{Conclusions}

The use of alternative TSSs is a widespread phenomenon in human genes, and by using exon array analysis, we identified nine genes with differential expression of isoforms between normal and tumor samples arising from differential TSS use. The changes observed for CHEK1, OSBPL1A and TCF12 were confirmed in several other cancer types indicating that the change in TSS usage is a general mechanism in cancer biology. The shift in OSBPL1A isoform ratio was experimentally shown to be regulated by the Wnt pathway, which is deregulated not only in CRC, but also in many other cancer types. A TCF12 short variant was found to be de novo synthesized in colorectal tumors, and TCF12 protein level was shown to be associated with progression free survival, further underlining the potential importance of alternative TSS usage in cancer development.

\section{Methods \\ Clinical samples}

One hundred and eight colorectal patient biopsies comprising 24 adjacent normal mucosa samples, 49 MSS adenomas (16 tubular, 12 tubulovillous and 21 villous), 30 MSS carcinomas (one stage I, 16 stage II and 13 stage III) and five MSI carcinomas (four stage II and one stage III) were examined (see Additional file 4 for a summary of the histopathological characteristics). All carcinomas were classified according to the WHO/UICC-TNM staging system. Immediately after surgery or polypectomy, the biopsies were embedded in Tissue-Tek O.C.T. Compound (Sakura Finetek), snap-frozen in liquid nitrogen and stored at $-80^{\circ} \mathrm{C}$. All patients gave informed written consent, and the study was approved by the Central Denmark Region Committee on Biomedical Research Ethics according to the Helsinki Declaration.

For recurrence free survival analysis, IHC was performed on a human colorectal TMA containing 268 biopsies from stage II adenocarcinomas. The fraction of patients with recurrence (distant metastasis excluding carcinosis) was 42 (16\%). The median duration of follow-up for the non-recurrence group was 1709 days (range 1099-1825 days), and for the recurrence group 770 days (range 95-1681). Before progression free survival analysis, 20 biopsies were excluded because the core was missing or lacked tumor material, leaving 248 samples for the analysis. A TMA containing 51 stage II adenocarcinomas and 50 normal mucosa samples was used to assess the expression of TCF12 and OSBPL1A.

\section{RNA preparation and Human Exon 1.0 ST Array labeling}

A Hematoxylin and Eosin stained cryostat section from all samples was used to evaluate tissue composition and when necessary macroscopic trimming was used to enrich the fraction of tumor cells to ensure a minimum of $60 \%$ neoplastic cells (median $85 \%(60 \%-90 \%)$ ). Total RNA was isolated from serial cryo-sections using the RNeasy Mini elute kit (Qiagen). Quality of the RNA was evaluated on the 2100 Bioanalyzer (Agilent), the median RNA Integrity Number (RIN) was 9.1 (6-10), and the median 28S/18S ribosomal peak ratio was 1.8 (1.1-3.1).

One hundred ng of total RNA was labeled according to the GeneChip Whole Transcript (WT) Sense Target Labeling Assay (Affymetrix, Inc., Santa Clara, CA) and hybridized to Human Exon 1.0 ST Arrays (Affymetrix, Inc., Santa Clara, CA) overnight. Scanning was performed in an Affymetrix GCS 3000 7G scanner. 


\section{Exon array data analysis}

Quantile normalization of exon array data and all further analysis was performed in the GeneSpring GX10 software (Agilent) using ExonRMA16 with core transcripts (17881 transcripts). For stabilization of variance, 16 was added to expression values before $\log 2$ transformation, which resulted in a minimum value of four in the $\log 2$ transformed dataset. Differential gene expression analysis was performed on transcript values based on core probe sets using Benjamini-Hochberg corrected unpaired t-tests. Alternative TSS analysis was limited to genes in the hg18 Refseq database containing two or more transcription start sites and at least one protein-coding isoform (2176 genes). To have probe sets in all exons of these genes, both core and extended probe sets of the exon array [35] were included in analysis of alternative TSS. Only genes for which more than $50 \%$ of the probe sets were expressed above background in at least half of both the normal and the tumor samples were included in the TSS analysis. Genes with potential alternative TSS or alternative splicing were identified using a multivariate ANOVA analysis (splicing ANOVA) and 663 genes had a splicing ANOVA pvalue $<0.05$ (Benjamini-Hochberg corrected). More stringent filtration on the $\mathrm{p}$-value $\left(<10^{-6}\right.$, Benjamini-Hochberg corrected) and the log2 splicing index (SI = probe set intensity/transcript expression value) $\mathrm{SI}>0.5$ or $\mathrm{SI}<-0.5$ resulted in 156 candidate genes. To identify genes expressing isoforms with alternative TSSs, the expression data of the candidate genes were visualized in a genomic context using the UCSC genome browser [36]. This manual curation identified nine candidate genes with apparent alternative TSS usage, which were further analyzed in two independent validation sample sets, and it was required that candidate genes were found to be significant in at least one of the validation sets (splicing ANOVA p-value $(<0.05)$ and SI $(>0.5$ or SI $<-0.5))$. The student's t-test was used when comparing two sample groups, except for the analysis of paired samples were a paired t-test was used.

\section{Quantitative real-time reverse-transcription PCR}

One $\mu \mathrm{g}$ of total RNA was converted to cDNA using a mixture of oligo(dT) and random nonamer primers and Superscript II Reverse Transcriptase (Invitrogen). Quantitative real-time reverse-transcription PCR (qRT-PCR) was performed in triplicates on a 7500 Fast or 7900 HT RealTime PCR System (Applied Biosystems). See Additional file 5 for the sequences of the primers used. Normalization was performed with UBC as previously described [37].

\section{Laser capture microdissection}

Laser capture microdissection was performed on cryosections from paired cancer and adjacent normal colon mucosa biopsies from six patients (two stage II and four stage III). Briefly, the sections were fixed in 95\% EtOH for $120 \mathrm{sec}$, followed by $15 \mathrm{sec}$ of staining in Arcturus Histogene Staining Solution (DFA Instruments), dehydrated in 95\% EtOH (30 sec) and 100\% EtOH (120 sec) before a final treatment in xylene for 120 sec. After drying of slides, epithelial and stromal cells were captured on individual caps using the Veritas 704 apparatus (Arcturus). Captured material was incubated with RLT buffer (Qiagen) for 20 minutes at room temperature in the presence of $30 \mathrm{mM} \beta$-mercaptoethanol, and, subsequently, RNA was extracted using RNeasy MinElute spin columns (Qiagen). Five ng RNA was used for cDNA synthesis using the Ovation PicoSL WTA System (NuGEN) followed by qRT-PCR as described above.

\section{TMA staining and scoring}

Staining of the TMAs was performed with the following antibodies; Anti-TCF12 (Catalog Number: 14419-1-AP, ProteinTech) in a 1:250 dilution and anti-OSBPL1A (Catalog Number: 18-202-335518, Genway Biotech) in a 1:400 dilution and indirect staining was used as previously described [38].

The TMAs were scored by two independent investigators using the VIS software (Visiopharm). The intensity of the staining was scored within the following categories (negative, 0; weak, 1; moderate, 2 and strong, 3) and the fraction of positive cancer cells (negative, 0; less than half, 1; $51-80 \%, 2$ and $>80 \%, 3$ ) was evaluated independently by two investigators. The agreement between the investigators was evaluated by Kappa statistics. STATA 9.2 software (StataCorp) was used to perform univariate analysis using the log rank test or the Cox proportional hazards model, for the Fishers exact test and for generating Kaplan-Meier plots.

\section{Wnt-pathway model system}

The colon cancer cell lines (Ls174T and DLD1) stably expressing inducible dominant-negative (dn)TCF1 or dnTCF4 were a kind gift from Dr. Hans Clevers (The Hubrecht Laboratory, The Netherlands), and have previously been described [39]. $\beta$-catenin knockdown was performed in DLD1 cells by transfecting with $20 \mathrm{nM}$ siRNA targeting $\beta$-catenin (Dharmacon) or $20 \mathrm{nM}$ scrambled nontargeting siRNA. Transfections were carried out using Lipofectamine 2000 (Invitrogen). RNeasy (Qiagen) was used for RNA extraction and random nonamer primers were used for cDNA synthesis. qRT-PCR was performed as described above.

\section{Transcription factor binding analysis}

For each of the selected genes, 300 bp upstream and $100 \mathrm{bp}$ downstream of the transcription start site were 
analyzed for transcription factor binding sites. Using the TOUCAN2 program [14], the regions were scanned for known binding domains from the Transfac Public v7 vertebrate database. Domains overrepresented relative to the Eukaryotic Promoter Database [40] were selected, and tumor and normal samples were compared.

\section{Additional Human Exon 1.0 ST datasets}

The external colon cancer validation dataset was downloaded from http://www.affymetrix.com and consisted of 18 paired samples of adenocarcinoma and adjacent normal tissue [35]. The internal independent colon cancer validation set consisted of nine adjacent normal biopsies, six tubular adenomas, 13 MSS cancer and 6 MSI cancer samples and has previously been described [29]. The bladder cancer dataset consisted of 11 samples of normal epithelium, 12 T1tumors and 12 T2-4 tumors [29]. Five additional datasets covering brain gliomas (GSE9385), gastric (GSE13195), lung (GSE12236), liver (GSE12941) and prostate cancer (GSE21034) were downloaded from the Gene Expression Omnibus. The brain glioma dataset contained 26 glioblastomas, 22 oligodendrogliomas and 6 control brain samples [41]. The gastric cancer dataset consisted of 44 paired samples of adenocarcinoma and adjacent normal tissue. The lung cancer dataset consisted of 40 paired normal and lung adenocarcinomas [42]. The liver cancer dataset contained 20 paired samples of hepatocellular carcinoma and adjacent nontumorous liver [43], and the prostate cancer dataset consisted of 29 normal adjacent benign samples, 131 primary tumors and 19 metastases [44].

\section{Bioinformatics analysis of differentially expressed protein features and mRNA regulatory elements}

First, the peptides corresponding to the unique sequences of the different isoforms were aligned against the Protein Data Bank database by PSIBLAST [45]. Then FeatureMap3D [46], PyMOL (Molecular Graphics System, Delano, Scientific system, LLC, Palo Alto, CA), and scripts created for the analysis were used to analyze and visualize the structures and/or conserved domains. Secondary structures were predicted by PSIpred [47]. Hydrophobicity plots were based on Kyte-Doolittle and HoppWoods scales to predict potential hydrophilic regions most likely exposed on the protein surface. NetPhos [18] was used to predict phosphorylation sites. Furthermore, signal peptide cleavage site prediction was performed [20] along with prediction of propeptide cleavage sites, Yin-Yang sites and N-glycosylation and O-glycosylation sites [48]. Protein functions that potentially change between alternative isoforms were compared with ProtFun [49]. Regulatory RNA elements in UTRs related to transcriptional and translational regulation were predicted by RegRNA [22,50,51].

\section{Additional material}

\section{Additional file 1: Expression of TCF12, OSBPL1A and TRAK1 in} paired normal and tumor samples

Additional file 2: qRT-PCR of laser capture microdissected samples

Additional file 3: OSBPL1A and TCF12 isoform expression in multiple cancer types

Additional file 4: Table with summary of the histopathological characteristics

Additional file 5: Sequence of primers used for qRT-PCR

\section{Acknowledgements}

The excellent technical assistance of Hanne Steen, Pamela Celis, Inge-Lis Thorsen Lisbet Kjeldsen, and Gitte Høj is gratefully appreciated. We thank Dr. Hans Clevers (The Hubrecht Laboratory, The Netherlands) for the inducible Ls174T-derived and DLD1- derived cell lines. Previously described dnTCF4 gene expression profiles were kindly provided by Laurens G. van der Flier (The Hubrecht Laboratory, The Netherlands). This work was supported by The John and Birthe Meyer Foundation, the EU project GENICA Grant number 201630, The Danish Council for Independent Research Medical Sciences, The Danish Council for Strategic Research, The Lundbeck Foundation, and The Danish Ministry of the Interior and Health.

\section{Author details}

'Department of Molecular Medicine, Aarhus University Hospital, Skejby, 8200 Aarhus N, Denmark. ²Department of Biostatistics and Computational Biology, Dana-Farber Cancer Institute, Harvard School of Public Health, Harvard University, Boston, MA 02115, USA. ${ }^{3}$ Department of Radiology, Harvard Medical School, Harvard University, Boston, MA 02115, USA. ${ }^{4}$ Bioinformatics Research Centre (BiRC), Aarhus University, 8000 Aarhus C, Denmark. ${ }^{5}$ Department of Signal Processing, Tampere University of Technology, P.O. Box 527, Fl-33101 Tampere, Finland. ${ }^{6}$ Department of Surgery P, Aarhus University Hospital, THG, 8000 Aarhus C, Denmark.

\section{Authors' contributions}

KT carried out the exon array analysis, the GRT-PCR validation, participated in the TMA scoring and wrote the manuscript. TS performed the Wnt model experiments. $B \varnothing$ carried out the selection of samples and extraction of RNA. MHR carried out the laser capture microdissection and CDNA synthesis and contributed to writing the manuscript. SV implemented the TF binding analysis. KW performed all in silico protein predictions. KQH stained the tissue sections and participated in the TMA scoring. PL and JSP assisted with the data analysis. AE constructed the TMAs. FM participated in the protein analysis. KL and CW created the refseq gene lists and assisted with data analysis. SL provided patient material and clinical data. LD assisted in exon array analysis. TFO and CLA participated in the design and coordination and participated in the writing of the manuscript. All authors read and approved the final manuscript.

Received: 4 April 2011 Accepted: 14 October 2011

Published: 14 October 2011

\section{References}

1. Ferlay J, Shin HR, Bray F, Forman D, Mathers C, Parkin DM: Estimates of worldwide burden of cancer in 2008: GLOBOCAN 2008. Int J Cancer 2010.

2. Jass JR: Classification of colorectal cancer based on correlation of clinical, morphological and molecular features. Histopathology 2007 50(1):113-130.

3. Kruhoffer $M$, Jensen $J$, Laiho P, Dyrskjot $L$, Salovaara R, Arango D, Birkenkamp-Demtroder K, Sorensen FB, Christensen LL, Buhl L, et al: Gene expression signatures for colorectal cancer microsatellite status and HNPCC. Br J Cancer 2005, 92(12):2240-2248.

4. Bienz M, Clevers H: Linking colorectal cancer to Wnt signaling. Cell 2000, 103(2):311-320.

5. Shitashige $M$, Naishiro $Y$, Idogawa M, Honda $K$, Ono M, Hirohashi S, Yamada T: Involvement of splicing factor-1 in beta-catenin/T-cell factor-4-mediated 
gene transactivation and pre-mRNA splicing. Gastroenterology 2007, 132(3):1039-1054.

6. Thorsen K, Mansilla F, Schepeler T, Oster B, Rasmussen MH, Dyrskjot L, Karni R, Akerman M, Krainer AR, Laurberg $S$, et al: Alternative splicing of SLC39A14 in colorectal cancer is regulated by the Wnt pathway. Mol Cell Proteomics 2011, 10(1):M110 002998.

7. Kimura K, Wakamatsu A, Suzuki Y, Ota T, Nishikawa T, Yamashita R, Yamamoto J, Sekine M, Tsuritani K, Wakaguri $\mathrm{H}$, et al: Diversification of transcriptional modulation: large-scale identification and characterization of putative alternative promoters of human genes. Genome Res 2006, 16(1):55-65.

8. Davuluri RV, Suzuki Y, Sugano S, Plass C, Huang TH: The functional consequences of alternative promoter use in mammalian genomes. Trends Genet 2008, 24(4):167-177.

9. Baek D, Davis C, Ewing B, Gordon D, Green P: Characterization and predictive discovery of evolutionarily conserved mammalian alternative promoters. Genome Res 2007, 17(2):145-155.

10. Battey J, Moulding C, Taub R, Murphy W, Stewart T, Potter H, Lenoir G, Leder P: The human c-myc oncogene: structural consequences of translocation into the IgH locus in Burkitt lymphoma. Cell 1983, 34(3):779-787.

11. Marcel $V$, Hainaut $P$ : $p 53$ isoforms - a conspiracy to kidnap p53 tumor suppressor activity? Cell Mol Life Sci 2009, 66(3):391-406.

12. Xu CF, Brown MA, Chambers JA, Griffiths B, Nicolai H, Solomon E: Distinct transcription start sites generate two forms of BRCA1 mRNA. Hum Mol Genet 1995, 4(12):2259-2264.

13. Van de Wetering M, Castrop J, Korinek V, Clevers H: Extensive alternative splicing and dual promoter usage generate Tcf-1 protein isoforms with differential transcription control properties. Mol Cell Biol 1996, 16(3):745-752.

14. Aerts $S$, Van Loo P, Thijs G, Mayer H, de Martin R, Moreau Y, De Moor B: TOUCAN 2: the all-inclusive open source workbench for regulatory sequence analysis. Nucleic Acids Res 2005, 33(Web Server issue):W393-396.

15. Blache P, van de Wetering M, Duluc I, Domon C, Berta P, Freund JN, Clevers H, Jay P: SOX9 is an intestine crypt transcription factor, is regulated by the Wnt pathway, and represses the CDX2 and MUC2 genes. J Cell Biol 2004, 166(1):37-47.

16. Hatzis $P$, van der Flier $L G$, van Driel MA, Guryev V, Nielsen F, Denissov $S$, Nijman IJ, Koster J, Santo EE, Welboren W, et al: Genome-wide pattern of TCF7L2/TCF4 chromatin occupancy in colorectal cancer cells. Mol Cell Biol 2008, 28(8):2732-2744.

17. Van der Flier LG, Sabates-Bellver J, Oving I, Haegebarth A, De Palo M, Anti M, Van Gijn ME, Suijkerbuijk S, Van de Wetering M, Marra G, et al: The Intestinal Wnt/TCF Signature. Gastroenterology 2007, 132(2):628-632.

18. Blom N, Gammeltoft S, Brunak S: Sequence and structure-based prediction of eukaryotic protein phosphorylation sites. J Mol Biol 1999, 294(5):1351-1362.

19. la Cour T, Kiemer L, Molgaard A, Gupta R, Skriver K, Brunak S: Analysis and prediction of leucine-rich nuclear export signals. Protein Eng Des Sel 2004, 17(6):527-536.

20. Emanuelsson O, Brunak S, von Heijne G, Nielsen H: Locating proteins in the cell using TargetP, SignalP and related tools. Nat Protoc 2007, 2(4):953-971.

21. Bendtsen JD, Nielsen $H$, von Heijne G, Brunak S: Improved prediction of signal peptides: SignalP 3.0. J Mol Biol 2004, 340(4):783-795.

22. Huang $H Y$, Chien $C H$, Jen KH, Huang HD: RegRNA: an integrated web server for identifying regulatory RNA motifs and elements. Nucleic Acids Res 2006, 34(Web Server issue):W429-434.

23. Grillo G, Turi A, Licciulli F, Mignone F, Liuni S, Banfi S, Gennarino VA, Horner DS, Pavesi G, Picardi E, et al: UTRdb and UTRsite (RELEASE 2010): a collection of sequences and regulatory motifs of the untranslated regions of eukaryotic mRNAs. Nucleic Acids Res 2010, 38(Database issue) D75-80.

24. Finn RD, Mistry J, Tate J, Coggill P, Heger A, Pollington JE, Gavin OL, Gunasekaran P, Ceric G, Forslund K, et al: The Pfam protein families database. Nucleic Acids Res 2010, 38(Database issue):D211-222.

25. Carninci P, Sandelin A, Lenhard B, Katayama S, Shimokawa K, Ponjavic J, Semple CA, Taylor MS, Engstrom PG, Frith MC, et al: Genome-wide analysis of mammalian promoter architecture and evolution. Nat Genet 2006, 38(6):626-635.
26. Yamashita R, Sathira NP, Kanai A, Tanimoto K, Arauchi T, Tanaka Y, Hashimoto SI, Sugano S, Nakai K, Suzuki Y: Genome-wide characterization of transcriptional start sites in humans by integrative transcriptome analysis. Genome Res 2011.

27. Cheong J, Yamada Y, Yamashita R, Irie T, Kanai A, Wakaguri H, Nakai K, Ito T, Saito I, Sugano $S$, et al: Diverse DNA methylation statuses at alternative promoters of human genes in various tissues. DNA Res 2006, 13(4):155-167.

28. Yegnasubramanian $S, W u Z$, Haffner MC, Esopi $D$, Aryee MJ, Badrinath $R$, He TL, Morgan JD, Carvalho B, Zheng Q, et al: Chromosome-wide mapping of DNA methylation patterns in normal and malignant prostate cells reveals pervasive methylation of gene-associated and conserved intergenic sequences. BMC Genomics 2011, 12(1):313.

29. Thorsen K, Sorensen KD, Brems-Eskildsen AS, Modin C, Gaustadnes M, Hein AM, Kruhoffer M, Laurberg S, Borre M, Wang K, et al: Alternative splicing in colon, bladder, and prostate cancer identified by exon array analysis. Mol Cell Proteomics 2008, 7(7):1214-1224.

30. An Y, Zhou Y, Ren G, Tian Q, Lu Y, Li H, Li K, Su T, Xu B, Chen S, et al: Elevated expression of MGb2-Ag/TRAK1 is correlated with poor prognosis in patients with colorectal cancer. Int J Colorectal Dis 2011.

31. Wang D, Claus CL, Rajkumar P, Braunstein M, Moore AJ, Sigvardsson M, Anderson MK: Context-dependent regulation of hematopoietic lineage choice by HEBAlt. J Immunol 2010, 185(7):4109-4117.

32. Wang $D$, Claus $C L$, Vaccarelli $G$, Braunstein M, Schmitt TM, ZunigaPflucker JC, Rothenberg EV, Anderson MK: The basic helix-loop-helix transcription factor HEBAlt is expressed in pro-T cells and enhances the generation of T cell precursors. J Immunol 2006, 177(1):109-119.

33. Starr TK, Allaei R, Silverstein KA, Staggs RA, Sarver AL, Bergemann TL, Gupta M, O'Sullivan MG, Matise I, Dupuy AJ, et al: A transposon-based genetic screen in mice identifies genes altered in colorectal cancer. Science 2009, 323(5922):1747-1750.

34. Tsuchihara K, Suzuki Y, Wakaguri H, Irie T, Tanimoto K, Hashimoto S, Matsushima K, Mizushima-Sugano J, Yamashita R, Nakai K, et al: Massive transcriptional start site analysis of human genes in hypoxia cells. Nucleic Acids Res 2009, 37(7):2249-2263.

35. Gardina PJ, Clark TA, Shimada B, Staples MK, Yang Q, Veitch J, Schweitzer A, Awad $T$, Sugnet $C$, Dee $S$, et al: Alternative splicing and differential gene expression in colon cancer detected by a whole genome exon array. BMC Genomics 2006, 7:325.

36. Kent WJ, Sugnet CW, Furey TS, Roskin KM, Pringle TH, Zahler AM, Haussler D: The human genome browser at UCSC. Genome Res 2002, 12(6):996-1006.

37. Andersen $\mathrm{CL}$, Jensen $\mathrm{JL}$, Orntoft TF: Normalization of real-time quantitative reverse transcription-PCR data: a model-based variance estimation approach to identify genes suited for normalization, applied to bladder and colon cancer data sets. Cancer Res 2004, 64(15):5245-5250.

38. Andersen CL, Christensen LL, Thorsen K, Schepeler T, Sorensen FB, Verspaget HW, Simon R, Kruhoffer M, Aaltonen LA, Laurberg $S$, et al: Dysregulation of the transcription factors SOX4, CBFB and SMARCC1 correlates with outcome of colorectal cancer. Br J Cancer 2009, 100(3):511-523.

39. van de Wetering M, Sancho E, Verweij C, de Lau W, Oving I, Hurlstone A, van der Horn K, Batlle E, Coudreuse D, Haramis AP, et al: The beta-catenin/ TCF-4 complex imposes a crypt progenitor phenotype on colorectal cancer cells. Cell 2002, 111(2):241-250.

40. Schmid CD, Praz V, Delorenzi M, Perier R, Bucher P: The Eukaryotic Promoter Database EPD: the impact of in silico primer extension. Nucleic Acids Res 2004, 32(Database issue):D82-85.

41. French PJ, Peeters J, Horsman S, Duijm E, Siccama I, van den Bent MJ, Luider TM, Kros JM, van der Spek P, Sillevis Smitt PA: Identification of differentially regulated splice variants and novel exons in glial brain tumors using exon expression arrays. Cancer Res 2007, 67(12):5635-5642.

42. Xi L, Feber A, Gupta V, Wu M, Bergemann AD, Landreneau RJ, Litle VR, Pennathur A, Luketich JD, Godfrey TE: Whole genome exon arrays identify differential expression of alternatively spliced, cancer-related genes in lung cancer. Nucleic Acids Res 2008, 36(20):6535-6547.

43. Satow R, Shitashige M, Kanai Y, Takeshita F, Ojima H, Jigami T, Honda K, Kosuge T, Ochiya T, Hirohashi S, et al: Combined functional genome survey of therapeutic targets for hepatocellular carcinoma. Clin Cancer Res 2010, 16(9):2518-2528. 
44. Taylor BS, Schultz N, Hieronymus H, Gopalan A, Xiao Y, Carver BS, Arora VK, Kaushik P, Cerami E, Reva B, et al: Integrative genomic profiling of human prostate cancer. Cancer Cell 2010, 18(1):11-22.

45. Altschul SF, Madden TL, Schaffer AA, Zhang J, Zhang Z, Miller W, Lipman DJ: Gapped BLAST and PSI-BLAST: a new generation of protein database search programs. Nucleic Acids Res 1997, 25(17):3389-3402.

46. Wernersson R, Rapacki K, Staerfeldt HH, Sackett PW, Molgaard A: FeatureMap3D-a tool to map protein features and sequence conservation onto homologous structures in the PDB. Nucleic Acids Res 2006, 34(Web Server issue):W84-88.

47. Bryson K, McGuffin LJ, Marsden RL, Ward JJ, Sodhi JS, Jones DT: Protein structure prediction servers at University College London. Nucleic Acids Res 2005, 33(Web Server issue):W36-38.

48. Julenius K, Molgaard A, Gupta R, Brunak S: Prediction, conservation analysis, and structural characterization of mammalian mucin-type Oglycosylation sites. Glycobiology 2005, 15(2):153-164.

49. Jensen LJ, Gupta R, Staerfeldt HH, Brunak S: Prediction of human protein function according to Gene Ontology categories. Bioinformatics 2003, 19(5):635-642.

50. Hamilton TL, Stoneley M, Spriggs KA, Bushell M: TOPs and their regulation. Biochem Soc Trans 2006, 34(Pt 1):12-16.

51. Kato S, Sekine S, Oh SW, Kim NS, Umezawa Y, Abe N, YokoyamaKobayashi M, Aoki T: Construction of a human full-length cDNA bank. Gene 1994, 150(2):243-250.

\section{doi:10.1186/1471-2164-12-505}

Cite this article as: Thorsen et al:: Tumor-specific usage of alternative transcription start sites in colorectal cancer identified by genome-wide exon array analysis. BMC Genomics 2011 12:505.

\section{Submit your next manuscript to BioMed Central and take full advantage of:}

- Convenient online submission

- Thorough peer review

- No space constraints or color figure charges

- Immediate publication on acceptance

- Inclusion in PubMed, CAS, Scopus and Google Scholar

- Research which is freely available for redistribution

Submit your manuscript at www.biomedcentral.com/submit 